\title{
Energy-efficient continual learning in hybrid supervised-unsupervised neural networks with PCM synapses
}

\author{
S. Bianchi ${ }^{1 *}$, I. Muñoz-Martin ${ }^{1 *}$, G. Pedretti ${ }^{1}$, O. Melnic ${ }^{1}$, S. Ambrogio ${ }^{2}$ and D. Ielmini ${ }^{1}$ \\ Politecnico di Milano, Milan, Italy. ${ }^{2}$ IBM Research-Almaden, San Jose, CA, USA. *Contributed equally.

\section{E-mail: stefano1.bianchi@polimi.it}

\begin{abstract}
Artificial neural networks (ANNs) can outperform the human ability of object recognition by supervised training of synaptic parameters with large datasets. Contrarily to the human brain, however, ANNs cannot continually learn, i.e. acquire new information without catastrophically forgetting previous knowledge. To solve this issue, we present a novel hybrid neural network based on CMOS logic and phase change memory (PCM) synapses, mixing a supervised convolutional neural network (CNN) with bio-inspired unsupervised learning and neuronal redundancy. We demonstrate high classification accuracy in MNIST and CIFAR10 datasets (98\% and 85\%, respectively) and energy-efficient continual learning of up to $30 \%$ of non-trained classes with $83 \%$ average accuracy.
\end{abstract}

Continual learning. ANNs, like multi-layer perceptrons and CNNs, show high and stable accuracy for pattern and object recognition [1]. However, as presented in Fig. 1a for the sequential training of ' $\mathrm{A}$ ' and 'B' subsets in a standard $\mathrm{CNN}$, they lack the necessary 'plasticity' for learning continually [2]. To overcome this limitation, also known as "stability-plasticity dilemma", we propose a novel neural network based on stable trained convolutional filters and bio-inspired unsupervised spike-timing-dependent plasticity (STDP), Fig. 1b [3].

Supervised-unsupervised network. Fig. 2a shows the main blocks of our architecture: (i) the CNN, (ii) the combinational logic and (iii) the unsupervised winner-take-all (WTA) network. First, the CNN is trained with a subset of input patterns, called trained classes, to develop the filters by offline supervised training. Then, the whole network is operated, where also new patterns, called non-trained classes, can be learnt and classified thanks to the unsupervised network (Fig. $2 \mathrm{~b}$ ). In the $\mathrm{CNN}$, input images are convolved with $20 \times 20$ trained filters that are extracted from the first convolutional layer of the networks in Fig. 3. Two types of filters are used, the class filters (Fig. 3a), each trained to recognize only a specific class of the dataset, and the feature filters (Fig. 3b), each specialized in extracting a certain feature [4]. The filters were trained with respect to a fixed threshold for directly mapping a specific feature. This generates a pattern of binary responses, which are equal to either $\mathrm{V}_{\mathrm{DD}}$, when the feature is found, or 0 , when the feature is not found. Fig. 4a shows the circuit used for convolution, exploiting the analogue inmemory matrix-vector multiplication (MVM) with PCM devices [5]. We always used 16 different filters, 7 class filters and 9 feature filters, resulting in a $4 \times 4$ feature map. Fig. $4 \mathrm{~b}$ shows the average feature maps for the MNIST dataset with 3 non-trained classes. These patterns have variable density P (i.e. active signals over total ones); thus, they must be equalized before the unsupervised block. Fig. $4 \mathrm{c}$ shows the equalization layer, i.e. a combinational logic with a $4 \times 4$ output pattern with $\mathrm{P}=25 \%$ (Fig. $4 \mathrm{~d}$ ). The logic gives higher priority to class filters. Finally, the equalized pattern feeds the WTA network with unsupervised learning via STDP and inhibitory synapses [3].

Implementation of PCM synapses. We used PCM synapses with one-transistor/one-resistor (1T1R) structure for both the $\mathrm{CNN}$ and the WTA. Fig. 5 shows the cumulative distributions of measured conductance for the PCM synapses of Fig. 4a. Here, 10 levels were programmed to serve as analogue weights for the MVM [5]. The reset (high resistance) state of the PCM was used as reference. Fig. 6 illustrates the cumulative distributions of the current in response to a class filter (a) and a feature filter (b). As expected, only one class shows a high current in response to a class filter. The non-trained classes only respond to feature filters, as the features are general enough to be found in both trained and non-trained classes. We tested an unsupervised WTA network with $4 \times 4$ patterns and 3 post-synaptic neurons (POSTs). Fig. 7 shows the equalized input patterns (a), the measured PCM conductances (b) and the POSTs firing activity (c). Presentation of two patterns in the first 500 'epochs', i.e. input appearance every $10 \mathrm{~ms}$ (bioinspired) or faster $(10 \mu \mathrm{s})$ [6], results in STDP learning. The presentation of the third pattern in the next 500 epochs results in further unsupervised learning without forgetting the first two patterns. This supports the role of STDP for continual learning. MNIST dataset. Fig. 8a shows that higher testing accuracy is obtained for an increasing number of POSTs per non-trained class. This highlights the necessity of neuronal redundancy to classify patterns that have more than one feature map (Fig. 4b). Redundancy is indeed found in biological networks as a mean to support learning [7]. Fig. $8 \mathrm{~b}$ shows that the global accuracy decreases from the ideal value of $98 \%$ for full training to $93 \%$ with 3 non-trained classes. Considering non-trained classes 2 , 5, 7, CMOS logic and PCM variability, a further 4\% decrease is observed, as shown in the confusion matrix of Fig. 9.

Energy efficiency. To reduce the energy consumption per input presentation, we introduced a burst-mode current integration for STDP and reduced the POST fire pulse applied to the PCM synapses to $100 \mathrm{~ns}$ (Fig. 10a) [3]. Fig. 10b shows that the unsupervised block requires an average energy consumption of $10^{-12} \mathrm{~J}$ for current integration and a $10^{-10} \mathrm{~J}$ for the consequent firing, which is lower than the average energy for convolution $\left(10^{-7} \mathrm{~J}\right.$, Fig. 11). The non-trained classes require similar energy consumption in the MVM respect to the trained ones. The additional energy required by the non-trained patterns is given by the learning activity of STDP, which is however negligible respect to convolution, thus sustaining the energy-efficient approach to continual learning.

CIFAR10 dataset. We tested our network for continual learning with the CIFAR10 dataset. Fig. 12 shows the recognition accuracy, reaching an $85 \%$ for fully trained dataset and $82 \%$ for 2 non-trained classes. Fig. 13 demonstrates continual learning for the non-trained "airplane" class, highlighting the fire activity of the POSTs, the equalization step for the new class and the learning procedure by STDP. The filters give three combinations of responses which highlights the role of neuronal redundancy for continual learning.

Conclusions. We presented a mixed supervised-unsupervised neural network with PCM synapses for continual learning. Good accuracy was obtained for full testing (98\% for MNIST, $85 \%$ for CIFAR 10 ) and learning of up to $30 \%$ non-trained classes via bio-inspired concepts such as STDP and neuronal redundancy. This novel network combines accuracy of supervised architectures and plasticity of unsupervised protocols for life-long learning autonomous systems.

References. [1] K. He, et al., (ICCV), 1026 (2015). [2] M. McCloskey et al., Psyc. of learning and motivation 24, 109, 1989. [3] S. Ambrogio et al., VLSI, 2016, DOI: 10.1109/VLSIT.2016.7573432. [4] M.D. Zeiler et al., ECCV, 8689, 2014. [5] G. W. Burr et al., "Advances in Physics": X, vol. 2, pp. 89-124, 2017. [6] G. Pedretti et al., Sci. Rep., vol. 7, p. 5288, 2017. [7] Hiratani et al., PNAS, 115 (29), July 2018. 


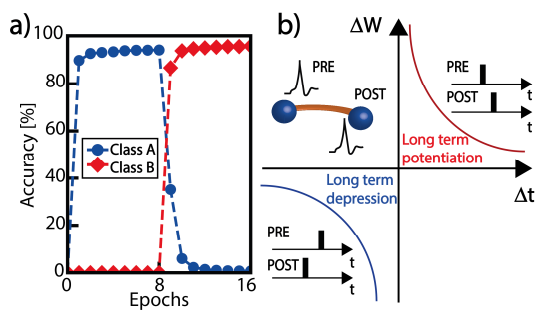

Fig. 1. (a) Catastrophic forgetting in ANNs: " $A$ " is no more recognized when " $B$ " is learnt. (b) Plastic behavior of STDP.

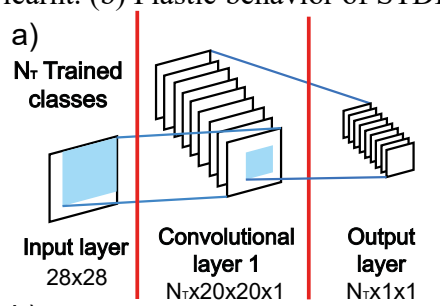

b)

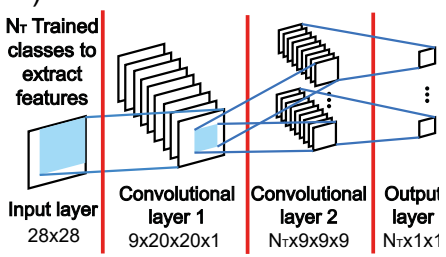

Fig. 3. Training methodology for extracting (a) $\mathrm{N}_{\mathrm{T}}=7$ "class filters" and (b) 9 "feature filters".

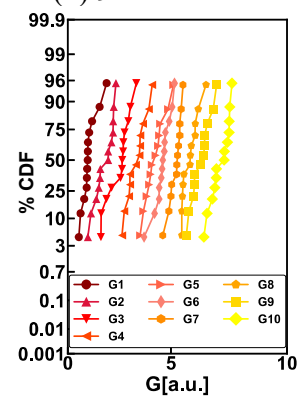

Fig. 5. Distributions of measured conductance levels of our PCMs.
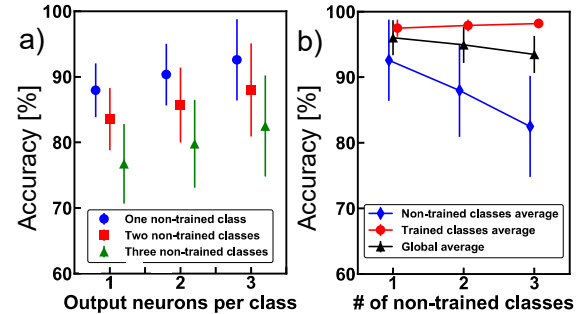

Fig. 8. (a) Neuronal redundancy in MNIST. (b) Global accuracy for increasing number of new classes and 3 output neurons per class.
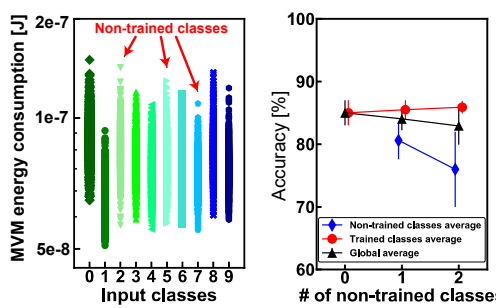

Fig. 11. Energy consumption per class in MVM.
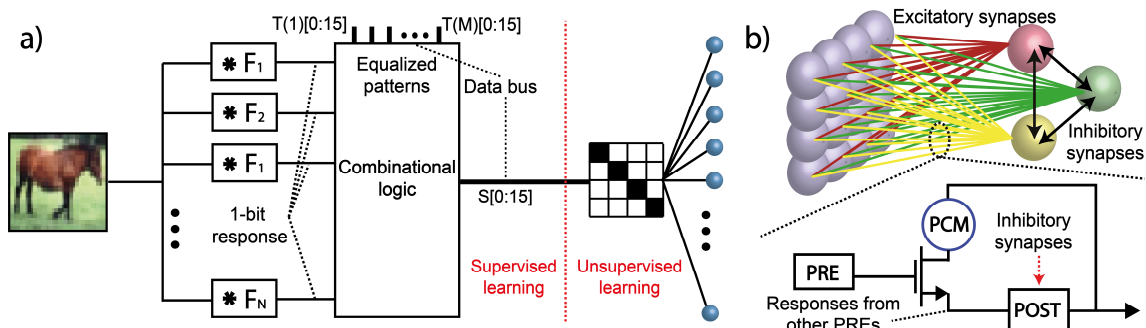

Fig. 2. Scheme of the network. (a) The input is convolved with $N$ filters, each giving a feature response. The responses select an equalized pattern from $\mathrm{T}(1)-\mathrm{T}(\mathrm{M}), \mathrm{M}$ is a generic number, that is then classified by STDP. (b) WTA classification by inhibitory and excitatory synapses.
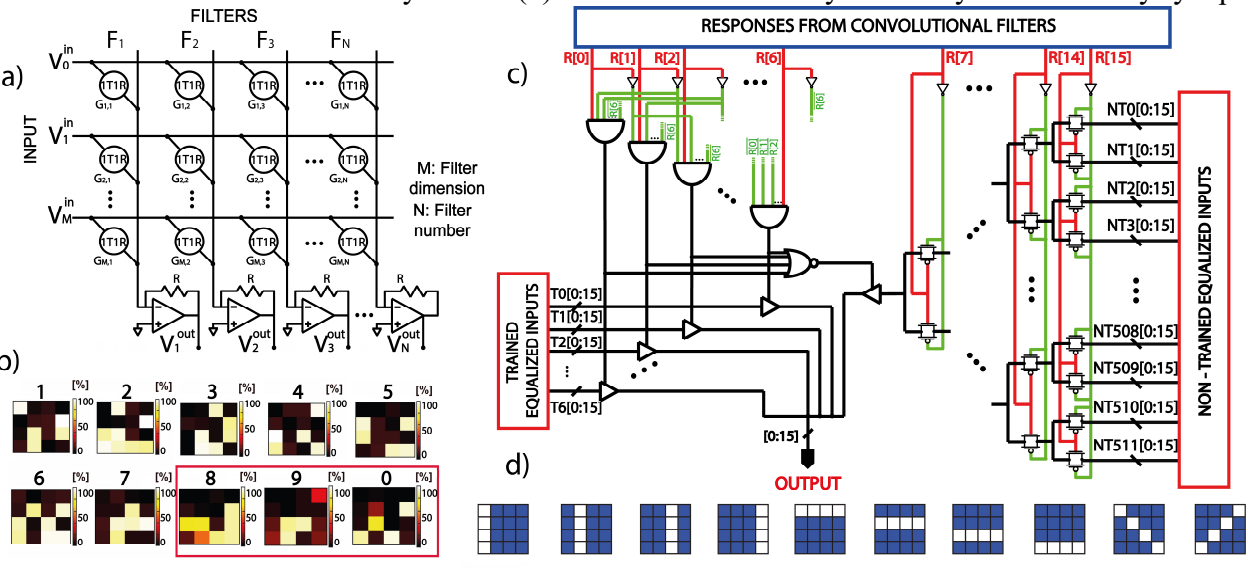

Fig. 4. (a) MVM circuit. (b) Average feature map outputs after MVM of MNIST trained and non-trained $(8,9,0)$ classes with the filters. (c) Implemented equalization circuit: signals R0-R6 come from class filters, the others from feature filters. (d) Example of equalized patterns ( $\mathrm{P}=25 \%)$ for STDP classification.

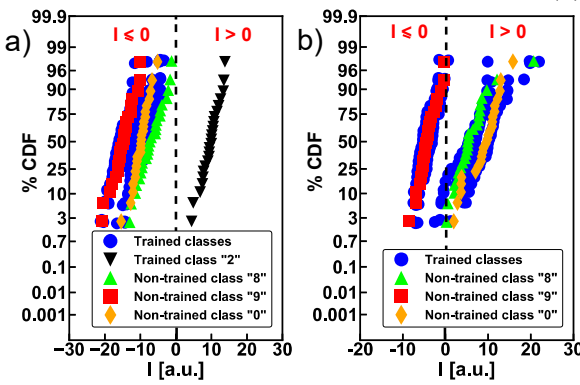

Fig. 6. Current distributions after MVM of trained and non-trained $(8,9,0)$ MNIST classes with a "class filter" (a) and a feature filter (b).

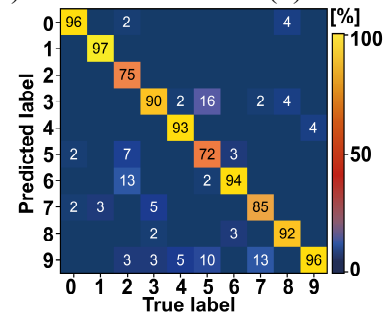

Fig. 9. Classification with full circuit implementation for continual learning of $2,5,7$.

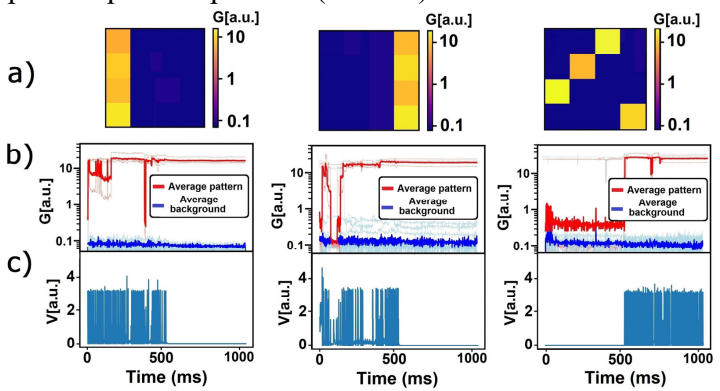

Fig. 7. (a) Experimental demo of continual learning with a new incoming pattern at 500 epochs. (b) Time evolution of pattern and background synapses. (c) POSTs spiking.

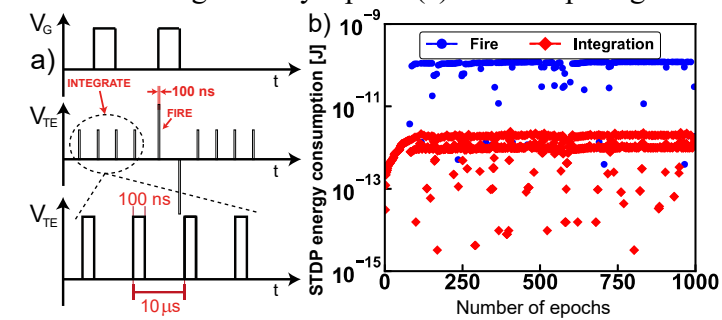

Fig. 10. (a) Burst-mode operation of STDP with 100 ns $V_{T E}$ pulses for integration power reduction. (b) Energy consumption in fire/integration during learning process.

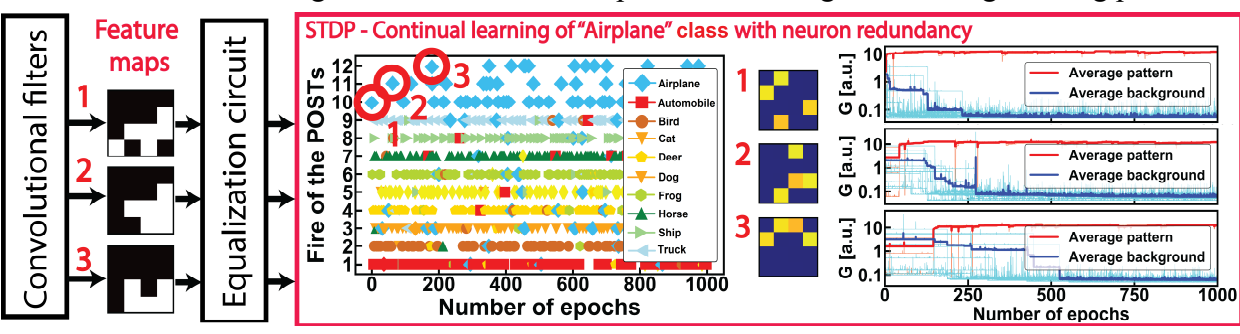

Fig. 13. Experimental demonstration of continual learning for the non-trained class "airplane" from CIFAR10. The new incoming class generates 3 different feature maps and thus 3 equalized patterns learnt by STDP. The POSTs spiking activities for all the classes highlight the neuronal redundancy. 